\title{
Marie-Françoise BASLEZ, Comment notre monde est devenu chrétien
}

Tours, CLD Éditions, 2008, 224 p.

\section{Pierre Lassave}

\section{(2) OpenEdition} Journals

Édition électronique

URL : http://journals.openedition.org/assr/21096

DOI : $10.4000 /$ assr.21096

ISSN : $1777-5825$

Éditeur

Éditions de l'EHESS

Édition imprimée

Date de publication : 31 décembre 2009

Pagination : $75-342$

ISBN : 978-2-7132-2218-4

ISSN : 0335-5985

Référence électronique

Pierre Lassave, "Marie-Françoise basLez, Comment notre monde est devenu chrétien », Archives de sciences sociales des religions [En ligne], 148 | octobre-décembre 2009, document 148-12, mis en ligne le 19 mai 2009, consulté le 21 septembre 2020. URL : http://journals.openedition.org/assr/21096 DOI : https://doi.org/10.4000/assr.21096

Ce document a été généré automatiquement le 21 septembre 2020.

(ㄷ) Archives de sciences sociales des religions 


\title{
Marie-Françoise BASLEZ, Comment notre monde est devenu chrétien
}

Tours, CLD Éditions, 2008, 224 p.

\author{
Pierre Lassave
}

\section{RÉFÉRENCE}

Marie-Françoise BASLEZ, Comment notre monde est devenu chrétien, Tours, CLD

Éditions, 2008, $224 \mathrm{p}$.

1 Signe d'un regain d'intérêt public pour les religions, les documentaires et les ouvrages de synthèse se multiplient aujourd'hui en France sur les origines du christianisme. Indiquons seulement le succès des émissions des essayistes Gérard Mordillat et Jérôme Prieur sur la chaîne Arte, les livres remarqués des historiens Paul Veyne (Quand notre monde est devenu chrétien, 2007) ou Bruno Dumézil (Les racines chrétiennes de l'Europe, 2005). L'ouvrage de Marie-Françoise Baslez s'inscrit dans cette dernière veine d'universitaires qui s'adressent à un plus large public qu'à l'habitude pour l'éclairer sur ce que la recherche dit sur des questions dont les réponses ne sont jamais définitives.

2 Comment, en effet, dans les trois premiers siècles de notre ère, le christianisme a-t-il pu passer d'une secte issue de Judée à la religion officielle de l'empire romain ? Le choix personnel de l'empereur Constantin en faveur de la religion chrétienne, en 312, a-t-il été aussi déterminant dans cette transformation que d'aucuns l'affirment (Veyne, par exemple) ? Ou s'agit-il plutôt, comme le montre l'historienne, de l'aboutissement d'un processus actif de tissage de communautés d'entraide associée à la formation d'un nouveau sens à donner à l'existence ?

Spécialiste des religions du monde gréco-romain et auteure remarquée de biographies sur saint Paul, Marie-Françoise Baslez tire ici une sorte de bilan d'une vingtaine d'années de recherches historiques et archéologiques mais aussi sociologiques sur un mouvement pluriel, indissociablement social et religieux, à l'origine de la formation des États occidentaux. 
Son titre, en forme de clin d'œil à l'essai de son aîné Paul Veyne, annonce bien l'intention d'explorer le "comment» de cette histoire avant d'en réduire le sens à quelque cause. C'est sur le terrain des sources scripturaires et épistolaires, des fouilles archéologiques, des chemins et des villes que le parcours des croyances peut ainsi s'appréhender dans sa diversité locale.

5 L'ouvrage remonte le temps en une dizaine de chapitres allant des premières manifestations chrétiennes, peu après la crucifixion de Jésus, jusqu'au fameux choix de Constantin. Malgré l'absence d'informations sur ces premières années, hormis les récits ultérieurs et rétrospectifs dans les épîtres de Paul ou les Actes de Luc, les historiens s'accordent sur un premier mouvement essaimant de Jérusalem à Rome en passant par Antioche et l'Asie Mineure, fidèles réunis par l'attente du retour imminent du Messie, sauveur du monde annoncé par les prophéties bibliques. Soulevant l'hostilité des autorités juives et romaines, ces communautés trouvent refuge au cœur des cités où elles entretiennent leur culte eschatologique tout en développant autour de leurs «maisonnées » tout un réseau d'assistance auprès des convertis, des indigents, des malades, des veuves et des orphelins.

En ces temps troublés de formation de l'empire à la jonction de peuples et de cultures forts différents, émergent les figures actives de " passeurs entre deux mondes » comme le philosophe judéo-grec Philon à Alexandrie ou l'historien judéo-romain Flavius Josèphe. Juif en même temps que citoyen romain, s'exprimant en grec, Paul appartient à cette veine de passeurs qui transforment le monde. À cet infatigable missionnaire revient l'idée révolutionnaire que l'on peut vivre son attente du Messie différemment selon que l'on est grec ou juif alors que, dans la tradition antique, être grec ou juif c'était différer radicalement par la langue et le culte. Par la distinction que l'apôtre instruit entre foi intérieure et tradition religieuse, une nouvelle voie s'entrouvre vers la participation des chrétiens aux droits et obligations de la Cité (« Rendez à César ce qui est à César »).

7 Au moment où le torchon brûle entre les autorités juives et romaines à Jérusalem (destruction du temple en 70), une telle libéralisation de la tradition ne va pas de soi. Non seulement elle suscite l'hostilité des pouvoirs en place, mais elle provoque la discorde au sein même des communautés qui reconnaissent le Christ comme messie. Ainsi peut-on comprendre la violence d'un livre comme l'Apocalypse de Jean tant contre «Babylone la prostituée » (Rome) que contre la « Synagogue de Satan », alias les chrétiens non juifs qui abandonnent, depuis Paul, les prescriptions impératives de la loi mosaïque (contrairement d'ailleurs à une tradition superficielle de lecture antijuive).

8 Passant rapidement sur ces divers conflits théologiques entre communautés concurrentes, déjà traités par de nombreux ouvrages, M.-F. Baslez met surtout l'accent sur l'engagement des maisons chrétiennes dans les questions de société et sur leur ancrage local. Le fameux « Il n'y a ni Juif ni Grec, ni esclave ni homme libre, ni homme ni femme » de l'épître aux Galates (Ga 3,28), loin d'être un slogan, transforme en profondeur les rapports sociaux. Aux figures masculines de passeurs viennent ainsi se joindre celles de "fortes femmes" comme Lydie, chef de famille qui prend la tête de l'Église de Philippes ou comme Thècle, disciple libre de Paul, devenue prophétesse et martyre. Outre l'émancipation des femmes chrétiennes, l'auteure aborde également la transformation des rapports entre citoyens libres et esclaves dans le sens de l'interdépendance des statuts. 
$9 \mathrm{Du} \mathrm{II}^{\mathrm{e}}$ au $\mathrm{III}^{\mathrm{e}}$ siècle, le mouvement de ceux qui se réclament du Christ progresse en adoptant une part des cultures dans lesquelles il s'installe. Les sources apocryphes attestent de la diversité des traditions qui en résultent, révoquant en doute la vision dualiste et anachronique qui oppose l'orthodoxie à l'hérésie (laquelle, comme l'on sait, signifiait à l'origine un choix légitime d'opinion). «Ainsi coexistent, au $\mathrm{II}^{\mathrm{e}}$ et $\mathrm{III}^{\mathrm{e}}$ siècles, un christianisme élitiste - qui réserve l'intégralité de la révélation à des inspirés, chez les montanistes, ou à des intellectuels mystiques, dans les cercles gnostiques - et un christianisme social, dans la tradition paulinienne, qui se donne pour objectif missionnaire d'agir là où l'on vit.» (p. 108). Mais comment, de ce christianisme éclaté, est-on passé à la future religion d'État ?

10 Trois facteurs s'associent à cet égard de façon déterminante à partir de la fin du $\mathrm{II}^{\mathrm{e}}$ siècle : primo, un travail intense d'unification doctrinale qui mobilise l'intelligentsia ecclésiale avec notamment la sélection progressive des textes canoniques et l'arrimage de la foi en Christ à la tradition biblique ; secundo, l'organisation des communautés de fidèles autour d'évêques localement influents et en relation de communication entre eux ; tertio, la manifestation de plus en plus publique de ce mouvement, notamment lors des moments de persécutions qu'il traverse au cours du $\mathrm{III}^{\mathrm{e}}$ siècle.

11 De l'édit de Caracalla (212) qui généralise la citoyenneté romaine à tout habitant libre de l'Empire à l'édit de Milan (313) qui reconnaît le christianisme comme religion licite, les rapports entre les pouvoirs religieux et politiques se recomposent en effet dans les larmes et le sang. Le gouvernement impérial des cultes oscille entre la restauration violente des traditions polythéistes anciennes, cause des pires moments de persécution des chrétiens sous Dèce et Dioclétien, et un hénothéisme pacificateur qui intègre la pluralité des croyances. Les communautés chrétiennes, en même temps qu'elles se détachent de leur souche judaïque, rencontrent la division entre un pôle notabiliaire local et un pôle charismatique itinérant, sans parler de l'émergence des retraits monastiques (que l'auteure aborde peu d'ailleurs). L'ère constantinienne résulterait, dès lors, de l'alliance entre les forces les plus stabilisatrices du moment. «En 312, les conversions de notables municipaux, l'intérêt des classes supérieures pour la question religieuse, les conversions de l'armée, rendaient ainsi possible, sinon probable, l'accession au pouvoir d'un chrétien ou d'un sympathisant » (p. 200).

12 Moins qu'un coup de génie qui change la face du monde, comme le suggère l'essai psychologisant de Veyne, le choix de Constantin serait l'aboutissement réaliste d'une prise d'influence morale et sociale d'un mouvement qui n'a pourtant pas, alors, le poids du nombre, d'une minorité fort active et organisée de 5 à $10 \%$ seulement des citoyens de l'Empire. Avant qu'une telle dynamique puisse prétendre à une quelconque religion d'État, il convient de noter que "l'originalité du christianisme est d'avoir fait sienne la notion de "religion", en travaillant sans cesse à créer du lien social, mais de lui donner un sens spécifique : le christianisme est la religion non plus d'un peuple, ni seulement d'un livre, mais aussi d'une Église, avec un principe unitaire indépendant de l'État. » (p. 193).

13 Au terme de son enquête sur les trois premiers siècles, l'historienne craint de laisser au lecteur l'image déconcertante d'une religion de petits groupes éclatés et différents, aussi loin de la marche triomphale vers la christianisation du monde que d'un spectaculaire fait du Prince. Mais son approche de la pénétration d'une religion par "capillarité dans le tissu social de la Cité » a au moins le mérite de réviser quelques idées reçues. Outre la remise en cause de la légende constantinienne, elle montre aussi 
comment les réseaux familiaux et associatifs, et l'exposition progressive de soi dans l'espace public, cadrent mal avec le mythe romantique d'une "Église des catacombes ". Le paradoxe est en définitive le fin mot de cette histoire : «Paradoxe d'une religion qui a toujours montré son sens de l'État, tout en affirmant pour la première fois le statut et les droits de la personne. Paradoxe d'une religion illégale, puis persécutée, qui acquit une meilleure visibilité dans la répression au lieu de disparaître. Paradoxe d'une religion mystique, que l'épreuve du martyre obligea à repenser son anthropologie en donnant une place au corps. Paradoxe d'une religion universaliste, mais capable, pour la première fois dans l'Antiquité, de dissocier culture et religion : on peut être chrétien en vivant en Grec comme en vivant en Juif. Paradoxe d'une religion unitaire, qui posa, la première, le principe de la liberté religieuse. Paradoxe, enfin, d'une religion qui procède d'une histoire globale du salut par le Christ, mais qui inscrivait son message dans des questions d'actualité. » (p. 206).

Cette brillante synthèse historique renvoie ainsi à la complexité d'un processus pluriel en s'appuyant notamment sur la sociologie dans la lignée épistémologique de l'École des Annales. L'ouverture interdisciplinaire élargit d'autant le questionnaire. On peut ainsi s'interroger sur la teneur sociologique et les formes prises par ces «associations relais » qu'évoque l'auteure à mi-chemin entre la maison familiale et l'Église institutionnelle. Croisent-elles, par exemple, des relations de métier ou d'économie dans la Cité ? De même, peut-on se demander si le terme anachronique de «lobby » sert bien la clarification des choses. Mais il nous semble sortir là de l'épure. Outre quelques coquilles, sans doute dues à une écriture pressée par la conjoncture éditoriale (par exemple, deux notes redondantes sur la notion d'évergétisme, p. 90 et p. 190), on peut regretter l'absence de cartes géographiques dans la mesure où le territoire s'impose comme une variable essentielle de cette histoire. 